\title{
O treino de controle do stress para hipertensos: uma contribuição para a medicina comportamental ${ }^{1}$
}

\author{
Marilda E.Novaes Lipp, ${ }^{2}$ Adriana B.Alcino, ${ }^{2}$ Márcia M.Bignotto ${ }^{2}$ e \\ Lúcia E.N.Malagris ${ }^{3}$
}

\begin{abstract}
Dentro do referencial da Medicina Comportamental, o presente estudo teve por objetivo verificar a eficácia de um treino de controle do stress na redução da reatividade cardiovascular de hipertensos, gerada pelo stress dos contactos interpessoais. A pressão arterial média de 20 sujeitos, hipertensos leves e moderados, foi aferida durante sessões experimentais que envolviam a necessidade de ser assertivo, antes e após o tratamento comportamental do stress. Os resultados indicaram que a maioria dos participantes era altamente inassertiva, tendo muito dificuldade de expressar tanto sentimetnos positivos como negativos, que o stress social é capaz de eliciar uma reatividade excessiva na pessoa hipertensa e que esta reatividade pode ser reduzida com o tratamento do stress. Palavras chaves: stress social, reatividade cardiovascular, assertividade, controle do stress, alexitimia, medicina comportamental.
\end{abstract}

\begin{abstract}
Stress management training of hypertensives: a contribution to behavioral medicine Within the Behavioral Medicine framework, the present study had for objective to verify the effectiveness of a stress management training in the reduction of cardiovascular reactivity of hypertensive subjects. The blood pressure of 20 borderline hypertensive individuals was measured during experimental sessions that involved the need to be assertive, before and after the behavioral treatment of stress. Results indicated that the majority of the participants were highly unassertive, that social stress is capable of eliciting excessive cardiovascular reactivity in hypertensive individuals and that this reactivity can be reduced by means of stress management treatment. Key words: social stress, cardiovascular reactivity, assertiveness, stress control, alexithymia, behavioral medicine.
\end{abstract}

A medicina comportamental é um campo multidisciplinar que combina métodos e resultados da pesquisa das ciências comportamentais e biomédicas. Novos avanços nas pesquisas experimentais que utilizam variáveis psicofisiólogicas são usados para ilustrar que os resul-

1. Esta pesquisa foi financiada pela FAPESP(Processo 92/22446) e pelo CNPq(Processo 530307/93-6)

2. Laboratório de Estudos Psicofisiológicos do Stress da Pontifícia Universidade Católica de Campinas.

3. Universidade Federal do Rio de Janeiro .

Endereço para correspondência: Dra. Marilda E.Novaes Lipp, Rua Tiradentes, 289, Conj. 91, Guanabara, CEP 13023190. Campinas, SP, Brasil, Telefone: (019) 234-0288. tados da ciência comportamental têm implicações para a área da medicina comportamental contemporânea (Epstein, 1992). Uma das questões mais fundamentais e intrigantes que os cientistas comportamentais enfrentam é selecionar quais estratégias de mudança de comportamento podem contribuir para o tratamento e prevenção de patologias.

Os distúrbios cardiovasculares estão entre os vários problemas de saúde que dependem tanto da herança familiar quanto dos hábitos de vida. A prevenção hoje em dia tem um papel decisivo para o controle de tais problemas (Casal e Cabala, 1991). 
O Treino de Controle do Stress para o Hipertenso(Lipp,1992) é um procedimento que se enquadra no âmbito da Medicina Comportamental, como método de controle e prevenção de respostas desadaptadas ao stress que podem desencadear reações fisiológicas, como aumentos da pressão arterial .

Os aumentos da pressão arterial, causados por momentos de intenso stress, designados como reatividade cardiovascular, podem ter um papel patofisiológico fundamental na ontogênese da hipertensão arterial essencial, conforme revisto por Matthews et al (1986) e Matthews et al(1993). A pressão arterial varia em todos durante o dia dependendo de vários fatores, mas não se sabe exatamente quais fatores determinam a magnititude destas mudanças. A teoria da reatividade prevê que a repetição de episódios discretos de hipertensão leva a adaptações estruturais que podem propiciar o estabelecimento da hipertensão arterial essencial. Tipicamente os estudos experimentais na área utilizam o método do "cold pressor"(Menkes et all,1989) ou teste de stress mental(Pickering e Gerin,1990) para evocarem a reatividade. Algumas críticas existem com relação ao uso destes testes, pois não se referem às vivências do dia a dia (Manuck et al, 1990). Alguns estudos tem objetivado aumentar a generalização dos estressores usados em situações de laboratório. Com este objetivo, um enfoque social tem sido acrescido ao estímulo utilizado para eliciar reatividade cardiovascular nos participantes, tal como entrevistas estressantes. Poucos trabalhos se referem aos efeitos do stress social na reatividade cardiovascular (Lipp,Bignotto e Alcino, 1997). Stress social é o termo usado para descrever a tensão que o ser humano pode sentir em função de contactos com outras pessoas quando ele necessita interagir de modo a expressar algum tipo de sentimento, expressar um desejo ou reivindicar seus direitos. Como o stress que o ser humano enfrenta mais frequentemente no seu dia a dia é justamente aquele oriundo dos contactos sociais, o estudo deste assunto torna-se de relevância para o tratamento não farmacológico da hipertensão arterial, essencial porque a literatura clínica(Morrisson et al,1985) indica que muitos pacientes hipertensos são caracterizados por deficits em rotular ou expressar afeto. Morrison (1989) sugere que essa dificuldade tem como consequência uma resposta fisiológica ao stress exacerbada.

Alguns estudos na área indicam que o treino de relaxamento, mental e físico, resulta em decréscimos terapêuticos na pressão arterial em repouso(Johnston, 1991), enquanto que outros sugerem que o relaxamento não é eficaz no controle pressórico(van Montfrans et al, 1990). Estes estudos utilizaram somente o treino de relaxamento como técnica de controle do stress. Não se encontrou na literatura investigações dos efeitos do relaxamento como parte de um treino de controle do stress mais completo, na reatividade cardíaca.

O presente estudo teve por objetivo verificar a eficácia do treino de controle do stress(Lipp, 1992) na redução da reatividade cardiovascular gerada pelo stress dos contactos interpessoais. Este estudo é uma contribuição da ciência do comportamento para a medicina comportamental.

\section{Método}

\section{Participantes}

Os participantes foram vinte adultos de classe sócio-econômica média-baixa, de 35 a 50 anos de idade, sendo 12 da raça branca e 8 da raça negra. Doze eram do sexo feminino e oito do sexo masculino. Os critérios para inclusão foram : ter pressão arterial entre 140x90 e 155 x $100 \mathrm{mmHg}$, comprovada não só no exame médico, mas também de acordo com o registro 
realizado por uma semana pelo próprio participante, não estar tomando medicação hipotensora e não ter problema renal, respiratório, diabetes ou patologia psiquiátrica comprovada.

\section{Material}

Nas sessões experimentais, se utilizou um Monitor de Pressão Arterial FINAPRESS, marca Ohmeda, modelo 2300, Denver, Colorado, que mede a pressão arterial continuamente. O Finapress é um equipamento que afere a pressão arterial, de modo não invasivo, através de uma pequena "cuff”, equipada com um fotopletismógrafo, que é colocada ao redor do dedo médio da mão não dominante do participante. Ao ser conectado ao computador, fornece a listagem das medidas registradas. Parati et al(1989) relataram uma grande covariância da pressão arterial do dedo com as medidas realizadas intra-arterialmente. Utilizou-se também um gravador para apresentação das descrições das cenas para o "role play" a fim, de se garantir a uniformidade dos estímulos estressores apresentados.

Os instrumentos utilizados na coleta de dados psicológicos foram :

Escala de Assertividade de Rathus (1973), composta por 30 itens que avaliam o nível de assertividade do respondente.

Três fitas com descrições das cenas provocadoras de stress. Elaboradas para uso em cada uma das sessões de eliciação de stress. Cada fita continha a descrição de 8 cenas e durava 1' em média por descrição. Três juízes avaliaram as cenas de cada fita que foram então reescritas até que houvesse concordância quanto a equivalência das mesmas no que se refere a eliciação da resposta de stress. Os sujeitos recebiam instrução para ouvirem a fita e responderem como o fariam na vida real.

Roteiro para as sessões de "role play". A situação de "role play" tinha por objetivo avaliar os efeitos imediatos que o stress social tem na pressão arterial de pessoas hipertensas. Após a descrição de cada uma das 8 cenas havia um período onde 4 interações verbais entre o paciente e o assistente de pesquisa ocorriam. As interações do assistente eram padronizadas e seguiam um roteiro, independentemente do que o participante dissesse. As duas primeiras cenas continham material bastante neutro e não exigiam assertividade por parte do sujeito. A seguir eram apresentadas 6 cenas que exigiam a expressão de assertividade, sendo duas positivas e 4 negativas, ou seja, em duas delas a pessoa precisaria aceitar um cumprimento ou fazer um elogio a alguém, enquanto que nas outras 4 ela precisaria se envolver em um confronto verbal e defender um direito seu, reclamar de algo ou alguém e expressar sentimentos negativos. As cenas foram baseadas em 6 dos elementos de Rathus (1975).

Material usado no tratamento do stress: O roteiro para tratamento de hipertensos de Lipp(1992) e Lipp e Rocha (1994) foi seguido. O treino se constituiu de 5 sessões de 2 horas e meia de duração, uma vez por semana, em grupos de 10 , onde os participantes receberam orientação comportamental sobre técnicas de controle do stress, inclusive como reconhecer os primeiros sinais de tensão, identificar as fontes de stress e utilizar técnicas cognitivo comportamentais para reduzir a resposta de excitação psicofisiológica do stress, como autoinstrução seguida de relaxamento e respiração profunda.

\section{Procedimento}

Após contactar o laboratório, os voluntários passavam pela avaliação cardiológica e eram encaminhados para a avaliação psicológica. Quando preenchiam os critérios de inclusão, assinavam um termo de consentimento e eram agendados para o experimento. 
Sessão Experimental de Reação ao Stress: Na sessão experimental, o participante sentava-se confortavelmente em uma poltrona reclinável e o manguito do FINAPRESS era instalado ao redor do dedo médio da sua mão não dominante. O equipamento, acoplado ao computador, era ligado. Após responder a quaisquer perguntas que surgissem, o procedimento a ser seguido era explicado em detalhes, a psicóloga aplicava os instrumentos de avaliação psicológica e se retirava por um período de $10^{\prime}$ de linha de base. Ao fim destes 10', a psicóloga retornava acompanhada de uma outra que iria atuar como interlocutora nas cenas de "role play". A partir dai, a primeira psicóloga não mais interagia com o participante. Ela tocava a fita, operava o computador e monitorava os dados cardiovasculares, enquanto a segunda sentava em frente ao paciente e interagia com ele de acordo com o roteiro pré-estabelecido. Ao término do "role play", as duas psicólogas se retiravam da sala e uma terceira linha de base de $10^{\prime}$ se seguia. As sessões experimentais, que duravam em média 60 minutos, foram todas individuais. A reatividade cardíaca de cada participante foi medida durante a linha de base inicial, a sessão de stress social e um período de linha de base final em cada sessão. Nas semanas seguintes à sessão experimental, os participantes recebiam, em grupo, o treino de controle do stress e eram instruídos a praticarem os exercícios de relaxamento e respiração profunda uma vez por dia. Em cada sessão, de grupo, era verificado se haviam feito uso das técnicas comportamentais ensinadas para lidarem melhor com o stress do dia a dia. A fim de garantir que as possiveis mudanças não fossem simplesmente devidas à pura passagem do tempo decorrida entre as sessões de stress ou devidas à uma maior familiaridade com o laboratório, independente- mente do treino de controle do stress, 10 participantes aleatoriamente escolhidos foram convidados a comparecerem ao laboratório uma segunda vez antes do treino de controle do stress ter início.

\section{Análise dos dados}

As unidades básicas de análise das medidas foram as médias da pressão arterial média durante os vários momentos. Uma vez que as respostas durante as cenas levavam em média 3 minutos somente os 3 últimos, minutos das linhas de base foram utilizados como medidas comparativas com as respostas durante as cenas.

\section{Medidas de reatividade}

A reatividade foi avaliada subtraindo-se a média da linha de base inicial das médias de cada situação experimental, isto é, o período em que os participantes escutavam a descrição de cada tipo de situação (neutra, negativa e positiva), durante as respostas que eles davam a cada tipo de cena e durante a última linha de base. As diferenças em reatividade, verificadas comparando-se as medidas durante as sessões antes e após o treino de controle do stress, foram analisadas através do teste t para grupos pareados.

\section{Medidas psicológicas}

Os níveis de assertividade dos participantes no final do tratamento foram comparados com os níveis iniciais, através do teste $\mathrm{t}$ bicaudal para amostras pareadas.

\section{Resultados e discussão}

\section{Medida de assertividade}

Avaliação dos resultados da aplicação da Escala de Assertividade de Rathus revelou que $75 \%$ dos sujeitos tinham dificuldades em expressarem sentimentos, defenderem seus 
direitos e opiniões. Eram portanto inassertivos, obtendo notas abaixo de zero na escala de assertividade. A entrevista psicológica e a observação durante as cenas do "role play" confirmaram o alto nível de inassertividade dos participantes. Em cenas onde era necessário reivindicar um direito(como um manual de uma $\mathrm{TV}$, que estava arbitrariamente sendo negado por um vendedor), muitos participantes aceitavam a recusa e se conformavam em ir embora sem o manual. Em outras cenas onde era necessário expressar agradecimento ou admiração, eles não sabiam o que dizer e muitas vezes diziam "ah, é difícil" ou "não sei o que dizer".

A inassertividade se manifestou tanto nas cenas positivas como nas negativas, confirmando o que Wolpe(1969) já havia sugerido, que a assertividade envolve os dois componentes. Como quase todos os participantes eram inassertivos e todos eram hipertensos, pode-se afirmar que existe uma coexistência entre as duas condições, porém sugere-se que estudos futuros controlem especificamente a variável assertividade, a fim de que se possa ter mais do que uma correlação simples da incidência de inassertividade e hipertensão arterial. Não houve diferença significativa antes e após o controle do stress no que se refere à assertividade. $\mathrm{O}$ fato de que o nível de assertividade dos participantes não mudou após o tratamento do stress não foi inesperado, uma vez que o tratamento não trabalhou com esta variável especificamente.

\section{Efeitos do stress social na pressão arterial}

A pressão arterial média do grupo variou durante a simulação, iniciando-se a 97,75 mm $\mathrm{Hg}$ na linha de base, elevando-se para $120,4 \mathrm{~mm}$ $\mathrm{Hg}$ durante as respostas às cenas em que tinham que expressar afeto e subindo 25,25 mm Hg durante as respostas onde havia necessidade de defender um direito. Durante a linha de base final, a pressão arterial média do grupo era, em média, 11,75 mmHG mais alta do que durante a linha de base inicial. A constatação de que a pressão arterial continuou alterada durante os 10 minutos de repouso ao fim do estudo, mostra o quanto o stress social pode afetar o hipertenso por períodos prolongados, mesmo após o estressor ter sido afastado. O fato de que a sessão de stress social eliciou uma alteração significativa na pressão arterial do grupo demonstra que a pessoa hipertensa responde ao stress das relações interpessoais com alterações na sua atividade cardiovascular. $\mathrm{O}$ fato de que essas alterações se prolongam, mesmo após 10 minutos de descanso na posição reclinada, pode ser interpretada como indicativa dos efeitos mais duradouros do stress sobre a atividade cardiovascular do paciente hipertenso. Ela também indica a demora dos hipertensos de se recuperarem do stress social e tem implicações clínicas tanto na área da Medicina como na da Psicologia. O processo psicoterápico de pacientes hipertensos deve ter como referencial o fato de que o stress interpessoal eleva a pressão arterial destes pacientes e de que este aumento de pressão permanece por um período considerável, a fim de se evitar danos maiores a estes pacientes. $\mathrm{O}$ aumento verificado durante o período em que a gravação da descrição das cenas era apresentada, atingiu o seu máximo durante o diálogo das cenas negativas, caiu um pouco durante as cenas positivas, vindo a diminuir durante o último período de descanso. Este efeito não pode ser atribuído ao simples fato de "estar falando", já que foi menor nas cenas consideradas neutras. A explicação mais coerente seria a de que o stress social gerado pela necessidade de ser assertivo gera uma reatividade cardiovascular nos hipertensos que perdura pelo menos por mais de 10 minutos. 


\section{Efeitos do treino de controle do stress na pressão arterial}

As mudanças ocorridas na pressão arterial média do grupo em função do treino de controle do stress podem ser vistas no Gráfico 1. A pressão arterial em todos os momentos após o treino de controle do stress foi significantemente menor do que antes do mesmo $(t=4,83, p=0,001)$. Além disto, os participantes conseguiram normalizar a pressão arterial durante os 10 minutos finais do estudo, mostrando que o treino de controle do stress é útil na redução da excitabilidade emocional durante o stress social.

O Gráfico 2 mostra as mudanças ocorridas em termos de reatividade cardiovascular. Pode-se verificar que em todos os momentos experimentais a reatividade ao stress social foi menor após o tratamento do stress do que antes $(\mathrm{t}=5,18, \mathrm{p}=0,002)$. Este efeito se manifesta também no período pós stress(últimos 10 minutos). Os dados demonstram que o treino de controle do stress é eficaz na redução da reatividade cardiovascular de hipertensos ao stress social e que auxilia também ao reduzir o tempo de recuperação após episódios de stress.

Os resultados evidenciam que a pura exposição da pessoa a estímulos socialmente aversivos não reduz a reatividade cardíaca, haendo necessidade de um tratamento especializado na área.

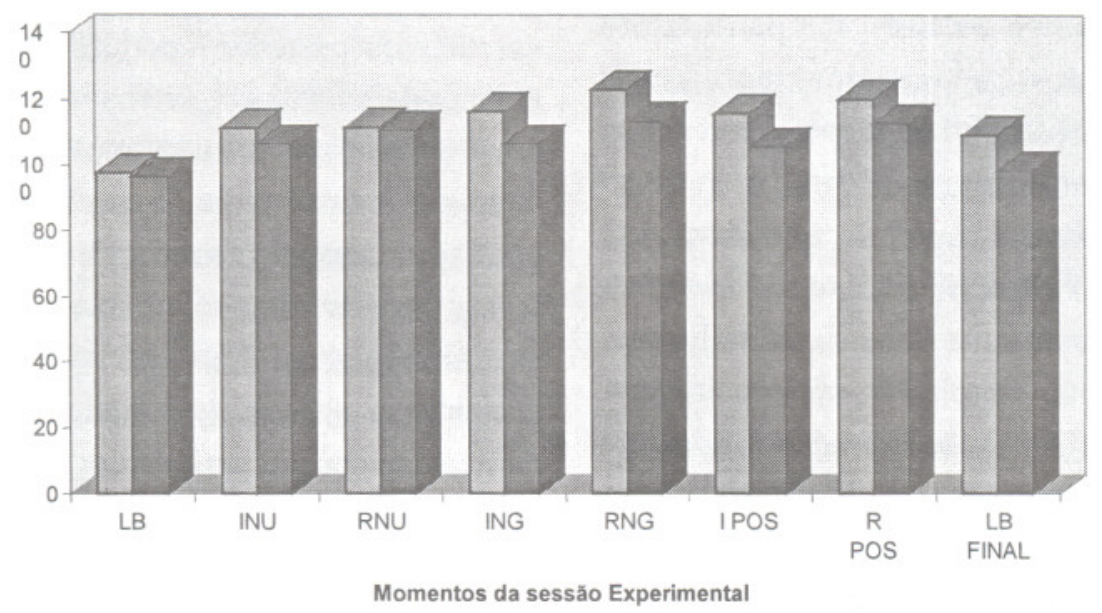

\section{Legenda:}

$\mathrm{LB}=$ Linha de Base Inicial

INU = Instruções das Cenas Neutras

RNU $=$ Respostas das Cenas Neutras

$\mathrm{ING}=$ Instruções das Cenas Negativas

RNG $=$ Respostas das Cenas Negativas

IPOS = Instruções das Cenas Positivas

LB FINAL $=$ Linha de Base Final

Gráfico 1. pressão arterial média antes e depois do treino de controle do stress Legenda 


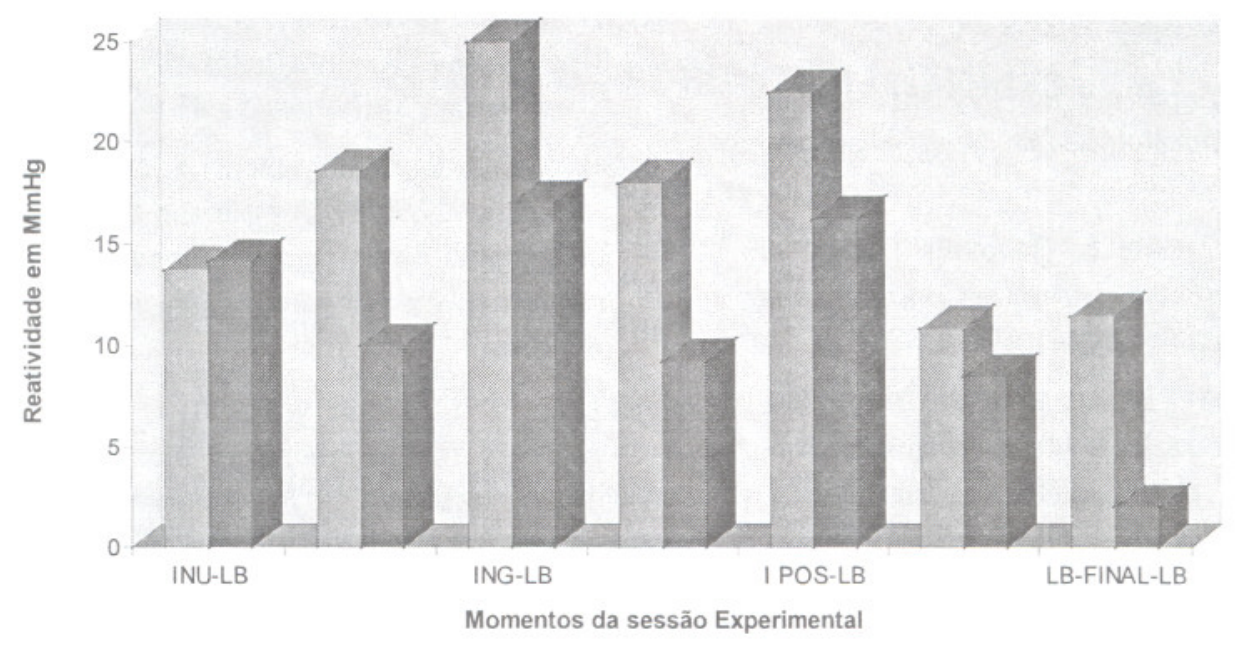

Legenda:

INU - LB = Instruções Neutras (média da reatividade cardiovascular durante as instruções neutras) me nos média da reatividade cardiovascular durante a primeira Linha de Base

ING - LB = Instruções Negativas menos Linha de Base

I POS - LB = Instruções Positivas menos Linha de Base

LB Final - LB = Linha de Base Final menos Linha de Base Inicial

Gráfico 2. Reatividade cardiovascular antes e depois do treino de controle do stress

\section{Conclusão}

A reatividade cardiovascular de pessoas hipertensas se mantém mesmo quando a interação social, o fato eliciador de stress social, se repete sem que elas tenham recebido um treino em como lidar com o stress, indicando que elas não se dessensibilizam para o evento, do ponto de vista cardiovascular, sòmente porque estão expostas a ele várias vezes. Cada vez que o evento estressor ocorre, o hipertenso reage com aumento da pressão arterial. O treino de controle do stress contribui para que essa reatividade seja reduzida. Considerando que a reatividade cardiovascular possa ser um indicador do funcionamento das artérias e um fator de risco para o desenvolvimento ou manutenção da hipertensão, estes dados tem implicações tanto para a Psicologia como para a Medicina, e mais especificamente para a área da Medicina Comportamental, uma vez que apontam para condições clínicas que podem ser controladas, pelo menos até certo ponto, através de uma intervenção psicológica adequada.

É recomendado que junto ao treino de controle do stress emocional se conduza também um treino de assertividade, a fim de se verificar o impacto de mudanças nesta característica na reatividade cardiovascular de hipertensos.

Recomenda-se tambem que outros estudos incluam um controle específico do nível de assertividade dos participantes, e que grupos de normotensos sejam incluídos.

Adicionalmente, interessante seria estudar experimentalmente a dificuldade de pessoas portadoras de hipertensão de expressarem afetos, condição mais comumente conhecida como alexitimia (Sifneous, 1972) e seu impacto nas elevações pressóricas em momentos de contactos sociais. Futuros trabalhos deverão tambem averiguar os efeitos do treino de controle do stress na pressão arterial basal dos hipertensos. 


\section{Referências Bibliográficas}

Casal, G. B. e Caballa,V.E. (1991)Manual de Psicologia Clínica Aplicada. Seglo Veintiuno Eels: Madrid.

Epstein, L. (1992)Role of Behavior Theory in Behavioral Medicine. Journal of Consulting and Clinical Psychology, 60 (4): 493-498.

Johnston, D. (1991) Stress Management in the Treatment of Mild Primary Hypertension. Hypertension, 17(4):III-63-67.

Lipp, M. N. (1992) O valor do Controle do Stress como terapêutica da Hipertensão Arterial.In M.Knobel(ed.) Psicossomática. (Pp.86-163). Campinas, São Paulo: NEP.

Lipp, M. N., Bignotto, M. M. e Alcino, A.B.(1997) Efeitos do treino de controle do stress social na reatividade cardiovascular de pacientes hipertensos. Teoria, Investigação e Prática. 2(1): 137146.

Lipp, M. N. e Malagris,L. N.(1996) Mitos e Verdades sobre o Stress. São Paulo:Ed.Contexto.

Lipp, M. N. e Rocha, J. C.(1994) Stress, Hipertensão Arterial e Qualidade de Vida. Campinas:Papirus.

Manuck, S. B.; Kasprowicz, A. L.; Muldoon, M. R. (1990) Behaviorally-evoked cardiovascular reactivity and hypertension: concepts issues and potential associations. Annals of Behavioral Medicine, 12:17-29.

Matthews, K. A., Weiss, T. M., Detre, T. Dembroski, T. M., Falkner, B. Manuck, S. B., Williams R. B. (eds) (1986) Handbook of Stress, Reactivity and Cardiovascular Disease. NY: John Wiley \& Sons.

Matthews, K.; Woodall, K. L. e Allen, M. T. (1993) Cardiovascular Reactivity to Stress Predicts Future Blood Pressure Status. Hypertension, 22:479-485.

Menkes, M. S.; Matthews; Krantz. D. Sincerely. (1989) Cardiovascular reactivity to the cold pressor test as a predictor of hypertension. Hypertension, 14, (5):524-530.

Morrison, R. L, Bellack, A. S, Manuck sincerely. B. (1985)Role of social competence in borderline essential hypertension. J Consult Clin Psychol, 53: 248-255.
Morrison, R. L. (1989) Psychometrics of SchallingSifneos and Toronto Alexithymia Scales. Psychometer Psychosom, 51:83-90.

Parati, G., Casadei, R., Groppelli, A., DiRienzo, M., e Mancia, G. (1989)Comparison of finger and intra-arterial blood pressure monitoring at rest and during laboratory testing. Hypertension, 13: 647-655.

Pickering, T. G, Gerin W.(1990) Cardiovascular reactivity in the laboratory and the role of behavioral factors in hypertension: A critical review. Ann Behavioral Med. 12: 3-16.

Rathus, S. A. (1973) A 30-item schedule for assessing assertive behavior. Behaviour Therapy, 4,398-406.

Rathus, S. A. (1975) Principles and Practices of Assertive Training. The Counseling Psychologist, 5 (4): 1975.

Sifneos, P. E. (1972) Short term psychotherapy and emotional crisis. Cambridge:Harvard UP.

Van Montfrans, G. A., Karemaker, j. M., Wieling, W. e Dunning, A. J. (1990) Relaxation therapy and continuous ambulatory blood pressure in mild hypertension:a controlled study. BMJ. 300:1368-1372.

Wolpe, J. (1969)The Practice of Behavior Therapy, NY:Pergamon Press. 